\title{
A Network Pharmacology Approach to Estimate Potential Targets of the Active Ingredients of Epimedium for Alleviating Mild Cognitive Impairment and Treating Alzheimer's Disease
}

\author{
Xianwei Gao, ${ }^{1,2}$ Shengnan Li, ${ }^{1}$ Chao Cong, ${ }^{1}$ Yuejiao Wang, ${ }^{1}$ and Lianwei Xu $\mathbb{D}^{1}$ \\ ${ }^{1}$ Longhua Hospital, Shanghai University of Traditional Chinese Medicine, Shanghai 200032, China \\ ${ }^{2}$ Shanghai University of Traditional Chinese Medicine, Shanghai 201203, China \\ Correspondence should be addressed to Lianwei Xu; doctorsherryxlw@163.com
}

Received 8 July 2020; Revised 21 December 2020; Accepted 15 January 2021; Published 28 January 2021

Academic Editor: José L. Rios

Copyright (c) 2021 Xianwei Gao et al. This is an open access article distributed under the Creative Commons Attribution License, which permits unrestricted use, distribution, and reproduction in any medium, provided the original work is properly cited.

Background. The present study made use of a network pharmacological approach to evaluate the mechanisms and potential targets of the active ingredients of Epimedium for alleviating mild cognitive impairment (MCI) and treating Alzheimer's disease (AD). Methods. The active ingredients of Epimedium were acquired from the Traditional Chinese Medicine System Pharmacology database, and potential targets were predicted using the TCMSP target module, SwissTargetPrediction, and PharmMapper database. Target proteins correlating with $\mathrm{MCI}$ and $\mathrm{AD}$ were downloaded from the GeneCards, DisGeNet, and OMIM databases. The common targets of Epimedium, MCI, and AD were identified using the Jvenn online tool, and a protein-protein interaction (PPI) network was constructed using the String database and Cytoscape. Finally, Gene Ontology (GO) and Kyoto Encyclopedia of Genes and Genomes (KEGG) enrichment analysis of the common targets was performed using DAVID, and molecular docking between active ingredients and target genes was modeled using AutoDock Vina. Results. A total of 20 active ingredients were analyzed, and 337 compound-related targets were identified for Epimedium. Out of 236 proteins associated with MCI and AD, 54 overlapped with the targets of Epimedium. The top 30 interacting proteins in this set were ranked by topological analysis. GO and KEGG enrichment analysis suggested that the common targets participated in diverse biological processes and pathways, including cell proliferation and apoptosis, inflammatory response, signal transduction, and protein phosphorylation through cancer pathway, MAPK signaling pathway, PI3K-Akt signaling pathway, HIF-1 signaling pathway, sphingolipid signaling pathway, FoxO signaling pathway, and TNF signaling pathway. Molecular docking analysis suggested that the 20 active ingredients could bind to the top 5 protein targets. Conclusions. The present study provides theoretical evidence for in-depth analysis of the mechanisms and molecular targets by which Epimedium protects against MCI, AD, and other neurodegenerative diseases and lays the foundation for pragmatic clinical applications and potential new drug development.

\section{Introduction}

The incidence of Alzheimer's disease (AD) is increasing significantly with the globally increasing average population age [1]. $\mathrm{AD}$ is an irreversible neurodegenerative disease and is the leading cause of dementia [2]. Forty-four million people worldwide currently suffer from dementia, and the number of afflicted people is expected to double by 2050 [3]. $\mathrm{AD}$ is thought to occur 20 years or more before symptoms appear, and then progressively deteriorate over time, resulting in the loss of physical function, disability, and death [4]. The pathogenesis of $\mathrm{AD}$ is closely related to epigenetic factors, including the environment, vascular disease, inflammation, oxidative stress, or other risk factors, whereas emblematic pathological manifestations include deposition of amyloid-protein (plaque) outside the brain neurons, and tau protein within the brain neurons [5]. These abnormal changes lead to neuronal injury or destruction and a sequence of complicated symptoms. Early symptoms include memory loss, cognitive impairment, apathy, and depression. As the disease progresses, the patient's memory and cognitive impairment gradually worsen, resulting in 
communication disorders, poor judgment, muddled thoughts, behavioral changes, aphasia, and difficulty swallowing and walking, which drastically interfere with the patient's daily life and impose a burden on the family $[6,7]$. The progression and symptoms of $\mathrm{AD}$ are continuous and unified, and patients usually transition from normal cognition to mild cognitive impairment (MCI) caused by $\mathrm{AD}$, which further develops into dementia [8]. Epidemiological studies have shown that approximately $10 \%-15 \%$ of people with amnesic-MCI morph into AD; in contrast, only $1 \%$ of healthy seniors develop AD [9].

MCI is a clinically recognized neurodegenerative disease that is defined by cognitive changes, and represents a transition stage between healthy aging and dementia [10]. This transition phase is considered to be an optimal treatment window for the protection of brain function, reduction of cognitive impairment, and delay of $\mathrm{AD}$, essentially gaining more time for patients with relatively good cognitive health [11]. Currently, the universal treatments for AD are divided into medication- and non-medication-based therapies. Non-medication therapies can maintain or improve the patient's quality of daily life, but cannot prevent further injury and destruction of neurons. Medication approved by the United States Food and Drug Administration (FDA) for therapeutic use, including rivastigmine, galantamine, donepezil, memantine, and memantine combined with donepezil, can ameliorate the patients' mental state and cognitive symptoms, and slow down memory loss, but the clinical efficacy varies from person to person and the treatment window is limited [12]. MCI is identified as a precursor to $\mathrm{AD}$; however, to date there is no explicit medication for the treatment of MCI and AD. Improving cognitive deficits before the onset of $\mathrm{AD}$ can reduce disease severity, and consequently, clinicians often prescribe cholinesterase inhibitors or memantine to alleviate symptoms $[13,14]$. Given this situation, the development of new drugs is essential for the effective treatment of MCI, $\mathrm{AD}$, and other neurodegenerative diseases.

Modern pharmacological studies have demonstrated that Epimedium is a flavonoid compound with estrogen-like effects and simultaneously possesses anti-aging, antioxidant, anti-apoptotic, and anti-inflammatory properties $[15,16]$. Estrogen protects and regulates mitochondrial function in the brain and plays a significant role in regulating neuroplasticity and synapse formation, with prominent potential for the treatment of AD [17]. These salient effects support the use of Epimedium for the prevention and treatment of various neurodegenerative diseases.

Epimedium, also known as Horny Goat Weed, is a perennial herbaceous plant that is widely applied in Asian countries, such as Japan, South Korea, and China $[18,19]$. Epimedium is principally used for curing impotence, infertility, amnesia, osteoporosis, functional diseases of the elderly, cardiovascular diseases, and primary ovarian insufficiency [20]. The Epimedium extract Icariin has been extensively studied in animal models and is able to reduce $\mathrm{A} \beta$ production and amyloid plaque deposition, as well as enhancing learning and memory capacity [21]. Icariin mediates the P13K/Akt pathway to inhibit oxidative stress of tau protein, reduce apoptosis, promote cell viability, and prevent oxidative damage in vitro [22]. Icariin, which is the dominant active ingredient of Epimedium, exerts protective effects on the nervous system. However, less research has been conducted on the other active ingredients of Epimedium.

Traditional Chinese Medicine (TCM) has the peculiarities of multiple ingredients, multiple targets, and multiple pathways, and using experimental methods to explain the potential mechanisms of a TCM can therefore be difficult. Network pharmacology is a new methodological system based on pharmacology and pharmacodynamics that has the potential to enable exploration of the efficacy of TCMs [23]. By analyzing complicated and multi-layered networks of the active ingredients, targets, and specific therapeutic effects of TCM using molecular biology and correlative databases, the synergistic interaction of multiple compounds and their molecular mechanisms can be elucidated. This method can improve our understanding of TCM and provides a new route for the development of new drugs based on active ingredients [24].

In the present study, we made use of network pharmacology to investigate the active ingredients, drug targets, and key pathways of Epimedium against $\mathrm{MCI}$ and $\mathrm{AD}$. The prediction of interactions between proteins implicated in MCI and $\mathrm{AD}$ and the active ingredients of Epimedium will highlight potential targets for further molecular studies, which may uncover the beneficial components of Epimedium and lead to the development of an effective treatment for neurodegenerative diseases. The experimental workflow is shown in Figure 1.

\section{Materials and Methods}

2.1. Identification of Active Ingredients in Epimedium. We searched for the active ingredients of Epimedium in the Traditional Chinese Medicine System Pharmacology (TCMSP, http://lsp.nwu.edu.cn/browse.php) database. The TCMSP contains comprehensive information about traditional Chinese herbs, including the chemical structure, oral bioavailability, drug-likeness, half-life, Caco-2 intestinal epithelial permeability, blood-brain barrier, and associated drug-target-disease network. We used pharmacokinetic properties, including absorption, distribution, metabolism and excretion (ADME), oral bioavailability $(\mathrm{OB}) \geq 30 \%$, and drug-likeness $(\mathrm{DL}) \geq 0.18 \%$, to screen the active ingredients of Epimedium. Compounds with $\mathrm{OB} \geq 30 \%$ have good absorption, and a $\mathrm{DL} \geq 0.18 \%$ is chemically conducive to drug development [25]. We then obtained the SDF structural formulae of active ingredients from the PubChem database (http://pubchem.ncbi.nlm.nih. gov/) for ulterior target prediction [26].

2.2. Target Prediction of Active Ingredients. We used the TCMSP target module, SwissTargetPrediction (http://www. swisstargetprediction.ch/), and PharmMapper (http://www. lilab-ecust.cn/pharmmapper/) databases to predict the potential targets of Epimedium active ingredients. The TCMSP database provides the chemical composition of Chinese herbs, as well as all-sided drug targets of active ingredients, 


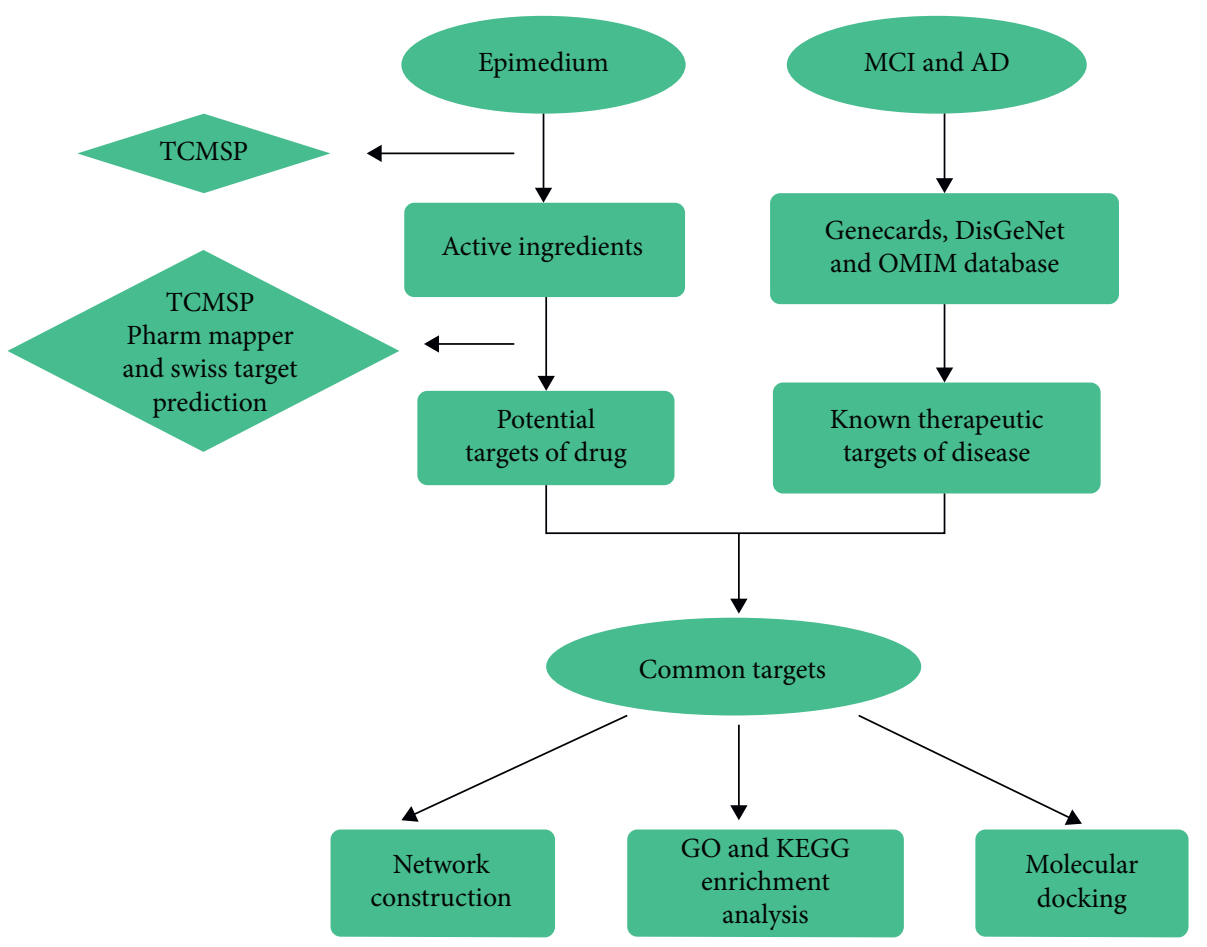

Figure 1: The experimental workflow for Epimedium against MCI and AD.

and their relation to diseases [25]. SwissTargetPrediction predicts the targets of active ingredients by comparing their structures to the two- and three-dimensional structures of known compounds [27]. PharmMapper is a freely accessible server that uses pharmacodynamic gene mapping methods to recognize candidate targets for drugs, natural products, or other newly found compounds with unidentified binding targets [28]. The resulting target protein names were ID mapped to their gene names using the UniProt database (http://www.UniProt.org/) [29].

2.3. Target Prediction of Disease. GeneCards (https://www. genecards.org/), DisGeNet (http://www.disgenet.org/), and OMIM (https://omim.org/) databases are regularly updated online tools containing information about human genes and genetic diseases. GeneCards includes proteomic, genetic, genomic, transcriptomic, clinical, and other information, which can be applied to predict human gene functions [30]. DisGeNet conveniently integrates the existing databases of mutation sites and human disease-related genes with corresponding information from a mass of literature to construct a unified gene or mutation site disease-related database [31]. OMIM is an authoritative database of human genes and genetic diseases [32]. In the present study, "mild cognitive impairment and Alzheimer's disease" were used as the keywords to collect known therapeutic targets from these three databases for the species "Homo sapiens."

2.4. Intersection between Active Ingredients and Disease Targets. We used the Jvenn online tool to generate a Venn diagram and identify common targets for Epimedium, $\mathrm{MCI}$, and AD. Common targets were submitted to String (https:// string-db.org/) to generate a protein-protein interaction (PPT) network [33]. The obtained network was visualized using Cytoscape v3. 7. 2, and topological analysis was performed using the network analysis module.

2.5. GO/KEGG Pathway Enrichment Analysis. GO is a constantly updated and internationally applicable gene function classification system that can be used to analyze functional enrichment of genes and proteins. KEGG pathway enrichment analysis provided a deeper explanation of different gene and protein functions by identifying enriched biochemical pathways in datasets. To gain insight into the gene functions and signaling pathways of the Epimedium targets, we used DAVID (https://david.ncifcrf.gov/), which is an online biological knowledge base and analysis tool, to identify enriched cellular components (CC), biological processes (BP), molecular function (MF), and KEGG pathways [34].

2.6. Validation of Putative Targets by Molecular Docking Analysis. Molecular docking is a burgeoning approach to explore drug mechanisms, elevate the pharmacological effect of drugs to the molecular level, and provide a theoretical basis for treating diseases. In the present study, the top five protein targets of Epimedium were selected based on PPI network analysis and included serum albumin (ALB), RACalpha serine/threonine-protein kinase (AKT1), interleukin-6 (IL6), caspase-3 (CASP3), and tumor necrosis factor (TNF), to perform molecular docking with the 20 active ingredients. We retrieved the SDFs of the 20 compounds from Pubchem 
(https://pubchem.ncbi.nlm.nih.gov/) and used Open Babel 2. 3. 2 software to convert SDFs into PDB files [26]. Next, we used PDB (http://www.rcsb.org/) to obtain the structural data of binding sites on AKT1 (PDB ID: 4EKL), ALB (PDB ID: 6JE7), CASP3 (PDB ID: 2J32), IL6 (PDB ID: 1ALU), and TNF (PDB ID: 5MU8) [35]. We used PyMOL 2. 3. 4 software to perform dehydration and ligand removal on the binding sites. The five proteins were modified using AutoDock Tools for hydrogenation and charge balancing. We used the "grid option" tool to set the grid point spacing to 1, adjust the volume of binding pocket so that the pre-docked molecules can rotate within the box in their most extended state, set the center of pocket as the center of binding site, and convert the protein and ligand small molecules into pdbqt format. Finally, we used AutoDock Vina 1. 1. 2 to perform molecular docking of the five proteins with the 20 ligand small molecules. We selected the receptors and ligands with strong binding free energy and generated three-dimensional graphs in PyMOL to analyze their interactions.

\section{Results}

3.1. Active Ingredients of Epimedium. A total of 130 chemical ingredients of Epimedium were obtained from the TCMSP database (Supplementary Table 1). After screening these and confirming their SDF structural formulae in Pubchem, the list was narrowed to 20 active ingredients of Epimedium. The characteristics of the 20 active ingredients are shown in Table 1.

3.2. Potential Targets of Active Ingredients. We searched the TCMSP database using the filtered list of Epimedium active ingredients and identified 204 compound-related targets. In addition, the SDF structural formulae of the active ingredients were used to search the SwissTarget and PharmMapper databases, and a list of relevant targets was acquired. The targets were screened to include only those with a probability value $\geq 0.5$ and a Normfit $\geq 0.8$. Using these cutoffs, 108 and 84 compound-related targets were obtained from SwissTarget and PharmMapper, respectively (Supplementary Table 2). After removal of the repeated targets, a total of 337 compound-related targets were identified (Figure 2).

3.3. Potential Targets of Disease. The GeneCards, DisGeNet, and OMIM databases were used to identify reviewed therapeutic targets in MCI and AD. The scores in GeneCards and DisGeNet were used to define a set of high-confidence interactions between these diseases and potential targets, with the relevance score $\geq 20$ in GeneCards and score-gda $\geq 0.1$ in DisGeNet. As a result, 546 AD-related targets and 441 MCI-related targets were acquired from GeneCards, and 303 AD-related targets and 5 MCI-related targets were obtained from DisGeNet. In addition, 523 AD-related targets and 85 MCI-related targets were obtained from OMIM. After removing the duplicate targets, 619 potential targets remained for MCI and 1166 for $\mathrm{AD}$, and 236 common targets for both diseases were identified (Supplementary Table 3).

3.4. Intersection of Active Ingredients and Disease Targets. A total of 54 targets overlapped for the Epimedium active ingredients, MCI, and AD. The overlap between the filtered targets was plotted using a Venn diagram (Figure 3). We used String to analyze the interactions between the 54 shared targets. The string analysis contained 54 nodes, 610 link edges, and 195 expected edges at the medium confidence. The average node degree was 22.6, the average local clustering coefficient was 0.737 , and the PPI enrichment $P$ value was $<0.001$. Cytoscape software was used to construct the PPI network. Different colors and circle sizes represent different biological information, with larger circles and darker colors (from orange to red) indicating stronger interactions (Figure 4(a)). In addition, we performed a topological analysis of the 54 common targets using a Cytoscape analysis module. The top 30 targets were ranked according to their degree values, represented by a bar graph (Figure 4(b)). Detailed topological analysis results and parameters are presented in Table 2.

3.5. GO/KEGG Function and Pathway Enrichment Analysis. To elaborate on the pivotal mechanisms underpinning the neuroprotective activity of Epimedium against MCI and AD, GO enrichment analysis was performed on 54 common targets using DAVID. The GO terms with $P$ values $<0.01$ were considered to be enriched (Supplementary Table 4). In the BP category, regulation of aging was enriched, associated with GO terms related to transcriptional regulation, regulation of cell proliferation and apoptosis, regulation of gene expression, inflammation, signal transduction, and protein phosphorylation (Figure 5(a)). In the CC category, target proteins were primarily distributed in the plasma membrane, and some in the cytosol and mitochondria (Figure 5(b)). In the MF category, target proteins were mainly involved in protein binding, with some involved in beta-amyloid binding (Figure 5(c)).

To identify pathways that are enriched, and therefore likely to be involved in the neuroprotective effects of Epimedium, we performed KEGG enrichment analysis of the 54 potential targets using DAVID, with a $P$ value cutoff of $<0.01$. We selected the top 20 enriched pathways, which were represented as bar and dot plots (Figure 6).

In addition, we identified targets that were shared between multiple pathways, which are listed in Supplementary Table 5. We constructed a target-pathway network, as shown in Figure 7(a). To understand the mutual effects of Epimedium, target, disease, and pathway, seven typical pathways were selected to set up an integral network, including the cancer pathway, mitogen-activated protein kinase (MAPK) signaling pathway, PI3K-Akt signaling pathway, HIF-1 signaling pathway, sphingolipid signaling pathway, FoxO signaling pathway, and TNF signaling pathway. Thus, an intricate network was formed to describe the effects of Epimedium active ingredients and potential targets of MCI and $\mathrm{AD}$ (Figure $7(\mathrm{~b})$ ). 
TABLE 1: The characteristics of active ingredients in Epimedium.

\begin{tabular}{|c|c|c|c|c|}
\hline Mol ID & Molecule name & Molecular formula & OB (\%) & $\mathrm{DL}$ \\
\hline MOL001510 & 24-epicampesterol & $\mathrm{C}_{28} \mathrm{H}_{48} \mathrm{O}$ & 37.58 & 0.71 \\
\hline MOL001645 & Linoleyl acetate & $\mathrm{C}_{20} \mathrm{H}_{36} \mathrm{O}_{2}$ & 42.10 & 0.20 \\
\hline MOL001771 & Poriferast-5-en-3beta-ol & $\mathrm{C}_{29} \mathrm{H}_{50} \mathrm{O}$ & 36.91 & 0.75 \\
\hline MOL001792 & DFV & $\mathrm{C}_{15} \mathrm{H}_{12} \mathrm{O}_{4}$ & 32.76 & 0.18 \\
\hline MOL003044 & Chryseriol & $\mathrm{C}_{16} \mathrm{H}_{12} \mathrm{O}_{6}$ & 35.85 & 0.27 \\
\hline MOL003542 & 8-lsopentenyl-kaempferol & $\mathrm{C}_{20} \mathrm{H}_{18} \mathrm{O}_{6}$ & 38.04 & 0.39 \\
\hline MOL000359 & Sitosterol & $\mathrm{C}_{29} \mathrm{H}_{50} \mathrm{O}$ & 36.91 & 0.75 \\
\hline MOL000422 & Kaempferol & $\mathrm{C}_{15} \mathrm{H}_{10} \mathrm{O}_{6}$ & 41.88 & 0.24 \\
\hline MOL004367 & Olivil & $\mathrm{C}_{20} \mathrm{H}_{24} \mathrm{O}_{7}$ & 62.23 & 0.41 \\
\hline MOL004373 & Anhydroicaritin & $\mathrm{C}_{21} \mathrm{H}_{20} \mathrm{O}_{6}$ & 45.41 & 0.44 \\
\hline MOL004382 & Yinyanghuo A & $\mathrm{C}_{25} \mathrm{H}_{24} \mathrm{O}_{6}$ & 56.96 & 0.77 \\
\hline MOL004384 & Yinyanghuo C & $\mathrm{C}_{20} \mathrm{H}_{16} \mathrm{O}_{5}$ & 45.67 & 0.50 \\
\hline MOL004386 & Yinyanghuo E & $\mathrm{C}_{20} \mathrm{H}_{16} \mathrm{O}_{6}$ & 51.63 & 0.55 \\
\hline MOL004391 & 8-(3-methylbut-2-enyl)-2-phenyl-chromone & $\mathrm{C}_{20} \mathrm{H}_{18} \mathrm{O}_{2}$ & 48.54 & 0.25 \\
\hline MOL004396 & 1,2-bis(4-hydroxy-3-methoxyphenyl) propane-1,3-diol & $\mathrm{C}_{17} \mathrm{H}_{20} \mathrm{O}_{6}$ & 52.31 & 0.22 \\
\hline MOL004425 & Icariin & $\mathrm{C}_{33} \mathrm{H}_{40} \mathrm{O}_{15}$ & 41.58 & 0.61 \\
\hline MOL004427 & Icariside A7 & $\mathrm{C}_{23} \mathrm{H}_{26} \mathrm{O}_{10}$ & 31.91 & 0.86 \\
\hline MOL000006 & Luteolin & $\mathrm{C}_{15} \mathrm{H}_{10} \mathrm{O}_{6}$ & 36.16 & 0.25 \\
\hline MOL000622 & Magnograndiolide & $\mathrm{C}_{15} \mathrm{H}_{22} \mathrm{O}_{4}$ & 63.71 & 0.19 \\
\hline MOL000098 & Quercetin & $\mathrm{C}_{15} \mathrm{H}_{10} \mathrm{O}_{7}$ & 46.43 & 0.28 \\
\hline
\end{tabular}

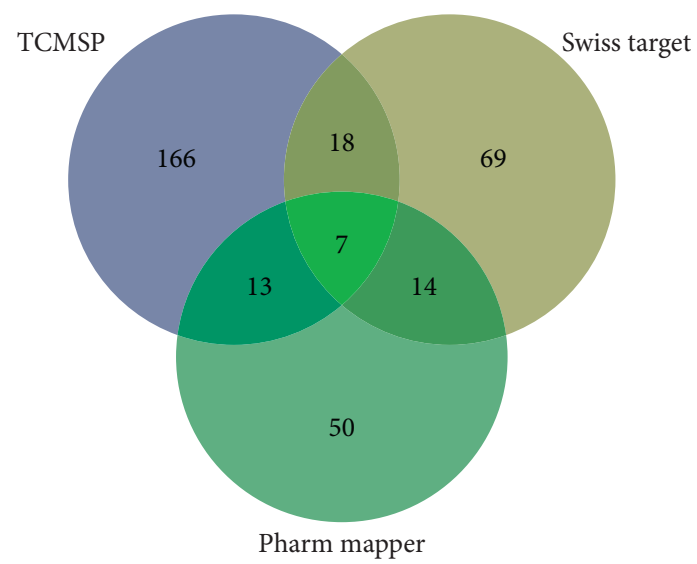

FIgUre 2: Analysis of potential targets of the active ingredients among TCMSP (blue), SwissTarget (yellow), and PharmMapper (green).

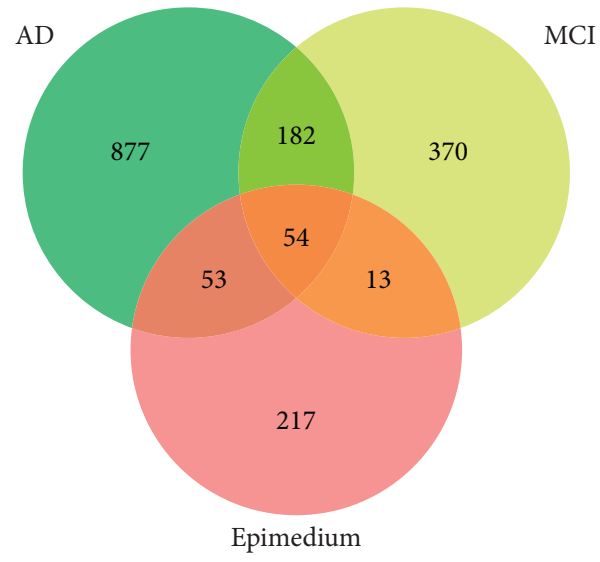

(a)

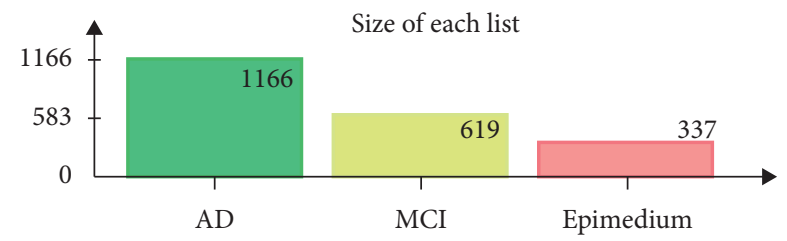

(b)

Figure 3: Analysis of predicted active ingredients-disease target genes among Epimedium, MCI, and AD. (a) Numbers of overlapped and specific genes among Epimedium, MCI, and AD. (b) Numbers of predicted genes from each set. 


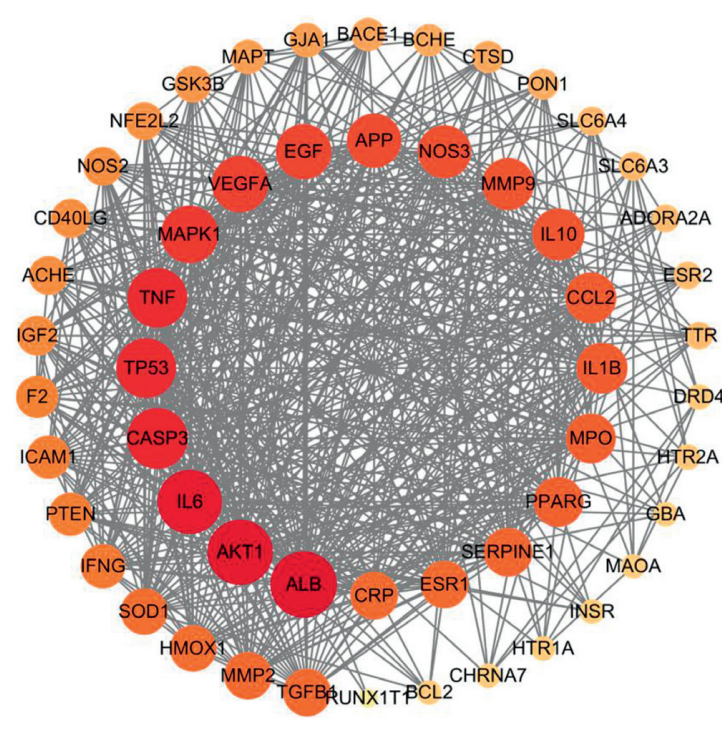

(a)

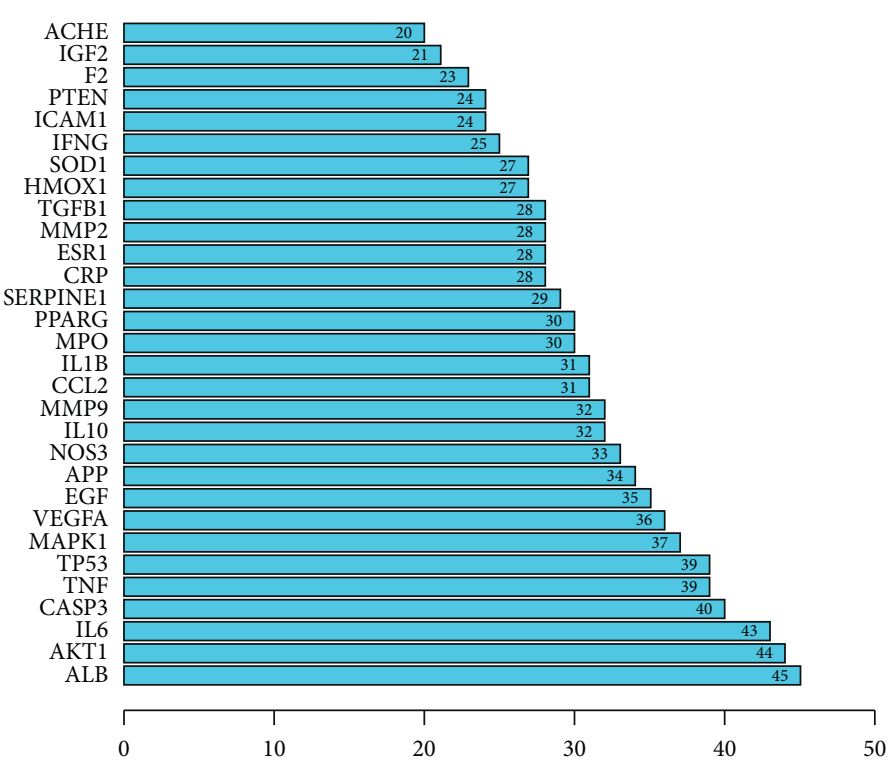

(b)

FIGURE 4: Protein-protein interaction (PPI) network of 54 target genes and top 30 targets from PPI network. (a) Visualization of PPI network of 54 target genes: from orange to red, the larger circle suggests stronger interactions. (b) Top 30 targets from PPI network.

3.6. Molecular Docking between Active Ingredients and Potential Targets. To validate the interactions between the screened Epimedium active ingredients and their proposed targets, we conducted molecular docking experiments. The binding free energy score and docking parameters are shown in Table 3. AutoDock Vina outputs the results in the form of an affinity energy, which is assessed by calculating the spatial effect, repulsion, hydrogen bonding, hydrophobic interactions, and molecular flexibility of the receptor-ligand complexes. The affinity energy is a prerequisite indicator to determine whether the ligand small molecule can effectively bind to the receptor, with lower energy values suggesting better binding capacity for the receptor and ligand. According to this scoring system, AKT1 and Yinyanghuo A, ALB and Yinyanghuo C, CASP3 and Yinyanghuo E, IL6 and Yinyanghuo $\mathrm{C}$, and TNF and Yinyanghuo A were selected for further analysis (Figure 8).

\section{Discussion}

In terms of TCM theory, MCI and AD fall under the category of amnesia and dementia. The same treatment for different diseases (Yi Bing Tong Zhi) is a fundamental treatment principle of TCM, which has accumulated vast experience in treating dementia over hundreds of years of clinical practice. Epimedium is one of the cross-sectional herbs that is prescribed as part of Chinese medicine, owing to its anti-apoptotic and anti-oxidative properties, and it is frequently applied to cure central nervous system diseases like forgetfulness, cognitive impairment, and dementia. The mechanism by which Epimedium exerts its therapeutic function is still poorly defined due to the complexity of its constituents. To enable better understanding of the mechanisms behind the protective effects of Epimedium, in the present study we made use of network pharmacology to explore multiple molecular perspectives, and to provide a theoretical basis for the treatment of neurodegenerative diseases.

We identified 20 chemical compounds as the potential active ingredients of Epimedium, many of which have been widely reported to possess inhibitory effects on neurological aging. For instance, icariin was used in Tg2576 mice (containing the Swedish AD mutant gene), with a standard dose of $60 \mathrm{mg} / \mathrm{kg}$. After three months of administration, the hippocampal nerve regeneration and working memory ability of the mice had improved [36]. Icariin also reduced the expression of amyloid $\beta 1-42$ and prevented the expression of amyloid precursor protein and $\beta$-site APP cleaving enzyme 1 in AD animal models [37]. Another active ingredient, kaempferol, inhibited the oxidative stress response and neuroinflammation in transgenic AD drosophila and an ovariectomized rat model, as well as reversing the loss of climbing ability and improving memory functions [38]. Quercetin is a flavonoid compound that protects neurons from oxidative and excitotoxic effects by modulating cell death mechanisms [39]. It also reduces $\mathrm{A} \beta$ polymerization, APP, and BACE1 expression and reduces oxidative stress [40]. Our molecular docking results predicted probable interactions between several active ingredients of Epimedium and protein targets that are related to MCI and AD. These predicted interactions imply that the active ingredients of Epimedium interact with proteins that have known functions in neurological diseases, which may explain its therapeutic effects. Based on these results, Epimedium and its active ingredients should be studied further, with a view to developing novel drugs for the treatment and prevention of MCI, AD, and other neurodegenerative diseases. 
TABLE 2: The topological analysis of 54 genes shared by Epimedium, MCI, and AD. Data were ranked by degree.

\begin{tabular}{|c|c|c|c|c|}
\hline Target & Name & Degree & $\begin{array}{l}\text { Betweenness } \\
\text { centrality }\end{array}$ & $\begin{array}{l}\text { Closeness } \\
\text { centrality }\end{array}$ \\
\hline ALB & Serum albumin & 45 & 0.061 & 0.869 \\
\hline AKT1 & RAC-alpha serine/threonine-protein kinase & 44 & 0.080 & 0.855 \\
\hline IL6 & Interleukin-6 & 43 & 0.045 & 0.841 \\
\hline CASP3 & Caspase-3 & 40 & 0.025 & 0.803 \\
\hline TNF & Tumor necrosis factor & 39 & 0.021 & 0.791 \\
\hline TP53 & Cellular tumor antigen p53 & 39 & 0.041 & 0.779 \\
\hline MAPK1 & Mitogen-activated protein kinase 1 & 37 & 0.039 & 0.757 \\
\hline VEGFA & Vascular endothelial growth factor A & 36 & 0.015 & 0.746 \\
\hline EGF & Pro-epidermal growth factor & 35 & 0.020 & 0.746 \\
\hline APP & Amyloid-beta precursor protein & 34 & 0.069 & 0.736 \\
\hline NOS3 & Nitric oxide synthase, endothelial & 33 & 0.015 & 0.726 \\
\hline IL10 & Interleukin-10 & 32 & 0.009 & 0.707 \\
\hline MMP9 & Matrix metalloproteinase- 9 & 32 & 0.006 & 0.707 \\
\hline CCL2 & C-C motif chemokine 2 & 31 & 0.005 & 0.697 \\
\hline IL1B & Interleukin-1 beta & 31 & 0.010 & 0.707 \\
\hline MPO & Myeloperoxidase & 30 & 0.009 & 0.697 \\
\hline PPARG & Peroxisome proliferator-activated receptor gamma & 30 & 0.008 & 0.688 \\
\hline SERPINE1 & Plasminogen activator inhibitor 1 & 29 & 0.005 & 0.679 \\
\hline CRP & C-reactive protein & 28 & 0.008 & 0.679 \\
\hline ESR1 & Estrogen receptor & 28 & 0.008 & 0.671 \\
\hline MMP2 & $72 \mathrm{kDa}$ type IV collagenase & 28 & 0.004 & 0.654 \\
\hline TGFB1 & Transforming growth factor beta-1 proprotein & 28 & 0.003 & 0.671 \\
\hline HMOX1 & Heme oxygenase 1 & 27 & 0.002 & 0.663 \\
\hline SOD1 & Superoxide dismutase $[\mathrm{Cu}-\mathrm{Zn}]$ & 27 & 0.013 & 0.671 \\
\hline IFNG & Interferon gamma & 25 & 0.001 & 0.631 \\
\hline ICAM1 & Intercellular adhesion molecule 1 & 24 & 0.000 & 0.624 \\
\hline PTEN & $\begin{array}{l}\text { Phosphatidylinositol 3,4,5-trisphosphate 3-phosphatase and } \\
\text { dual-specificity protein phosphatase }\end{array}$ & 24 & 0.005 & 0.624 \\
\hline $\mathrm{F} 2$ & Prothrombin & 23 & 0.010 & 0.639 \\
\hline IGF2 & Insulin-like growth factor II & 21 & 0.005 & 0.616 \\
\hline $\mathrm{ACHE}$ & Acetylcholinesterase & 20 & 0.031 & 0.609 \\
\hline CD40LG & CD40 ligand & 20 & 0.002 & 0.596 \\
\hline NOS2 & Nitric oxide synthase, inducible & 20 & 0.000 & 0.596 \\
\hline NFE2L2 & Nuclear factor erythroid 2-related factor 2 & 19 & 0.001 & 0.589 \\
\hline GSK3B & Glycogen synthase kinase- 3 beta & 18 & 0.001 & 0.596 \\
\hline MAPT & Microtubule-associated protein tau & 16 & 0.007 & 0.589 \\
\hline GJA1 & Gap junction alpha-1 protein & 16 & 0.000 & 0.570 \\
\hline BACE1 & Beta-secretase 1 & 15 & 0.002 & 0.582 \\
\hline CTSD & Cathepsin D & 14 & 0.003 & 0.570 \\
\hline BCHE & Cholinesterase & 14 & 0.016 & 0.564 \\
\hline PON1 & Serum paraoxonase/arylesterase 1 & 13 & 0.001 & 0.564 \\
\hline SLC6A4 & Sodium-dependent serotonin transporter & 11 & 0.014 & 0.546 \\
\hline SLC6A3 & Sodium-dependent dopamine transporter & 11 & 0.010 & 0.552 \\
\hline TTR & Transthyretin & 10 & 0.001 & 0.552 \\
\hline ESR2 & Estrogen receptor beta & 10 & 0.000 & 0.535 \\
\hline ADORA2A & Adenosine receptor $\mathrm{A} 2 \mathrm{a}$ & 10 & 0.001 & 0.546 \\
\hline HTR2A & 5-hydroxytryptamine receptor $2 \mathrm{~A}$ & 8 & 0.004 & 0.530 \\
\hline DRD4 & D (4) dopamine receptors & 8 & 0.003 & 0.530 \\
\hline BCL2 & Apoptosis regulator $\mathrm{Bcl}-2$ & 7 & 0.000 & 0.515 \\
\hline HTR1A & 5-hydroxytryptamine receptor $1 \mathrm{~A}$ & 7 & 0.001 & 0.473 \\
\hline INSR & Insulin receptor & 7 & 0.000 & 0.510 \\
\hline MAOA & Amine oxidase [flavin-containing] A & 7 & 0.001 & 0.417 \\
\hline GBA & Lysosomal acid glucosylceramidase & 7 & 0.000 & 0.505 \\
\hline CHRNA7 & Neuronal acetylcholine receptor subunit alpha-7 & 7 & 0.001 & 0.473 \\
\hline RUNX1T1 & Protein CBFA2T1 & 2 & 0.000 & 0.457 \\
\hline
\end{tabular}

In the present study, KEGG pathway and GO enrichment analysis was carried out for 54 common targets of Epimedium, MCI, and AD. Based on these analyses, we identified a subset of proteins, AKT1, MAPK1, CASP3, IL6, TNF, BCL2, TP53 and NOS3, which exert their effects via multiple different biochemical pathways. Such proteins are 


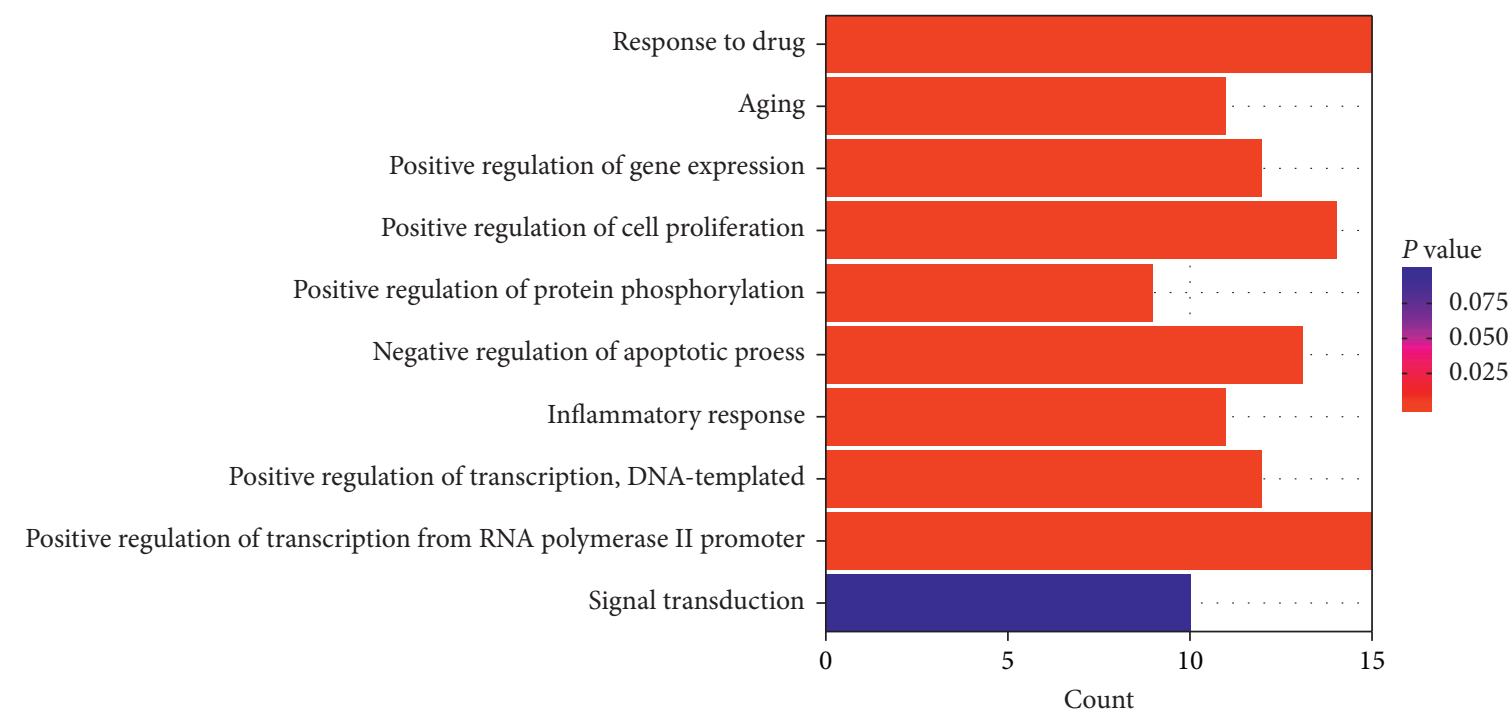

(a)

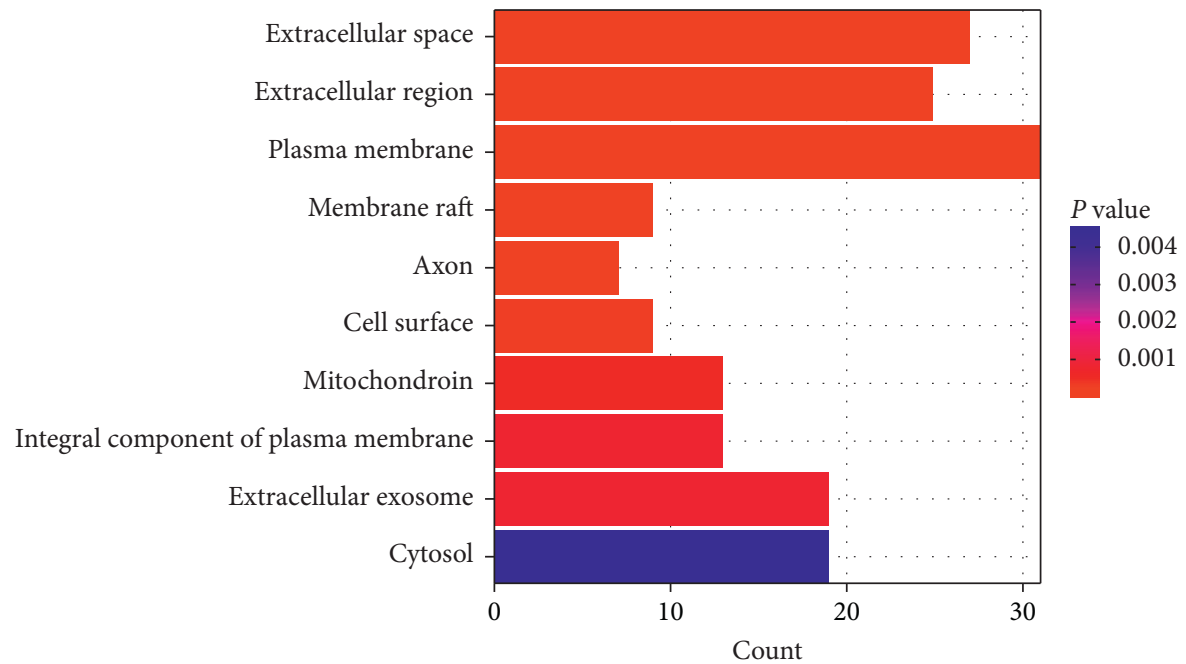

(b)

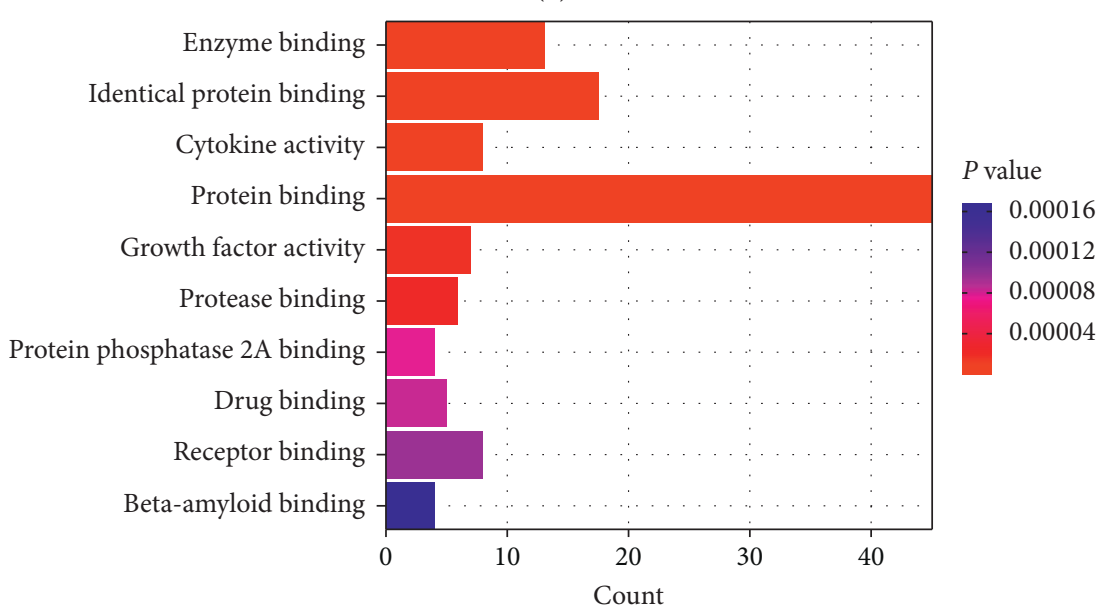

(c)

Figure 5: GO enrichment analysis of 54 target genes. (a) Biological process enrichment analysis. (b) Cellular component enrichment analysis. (c) Molecular function enrichment analysis. 


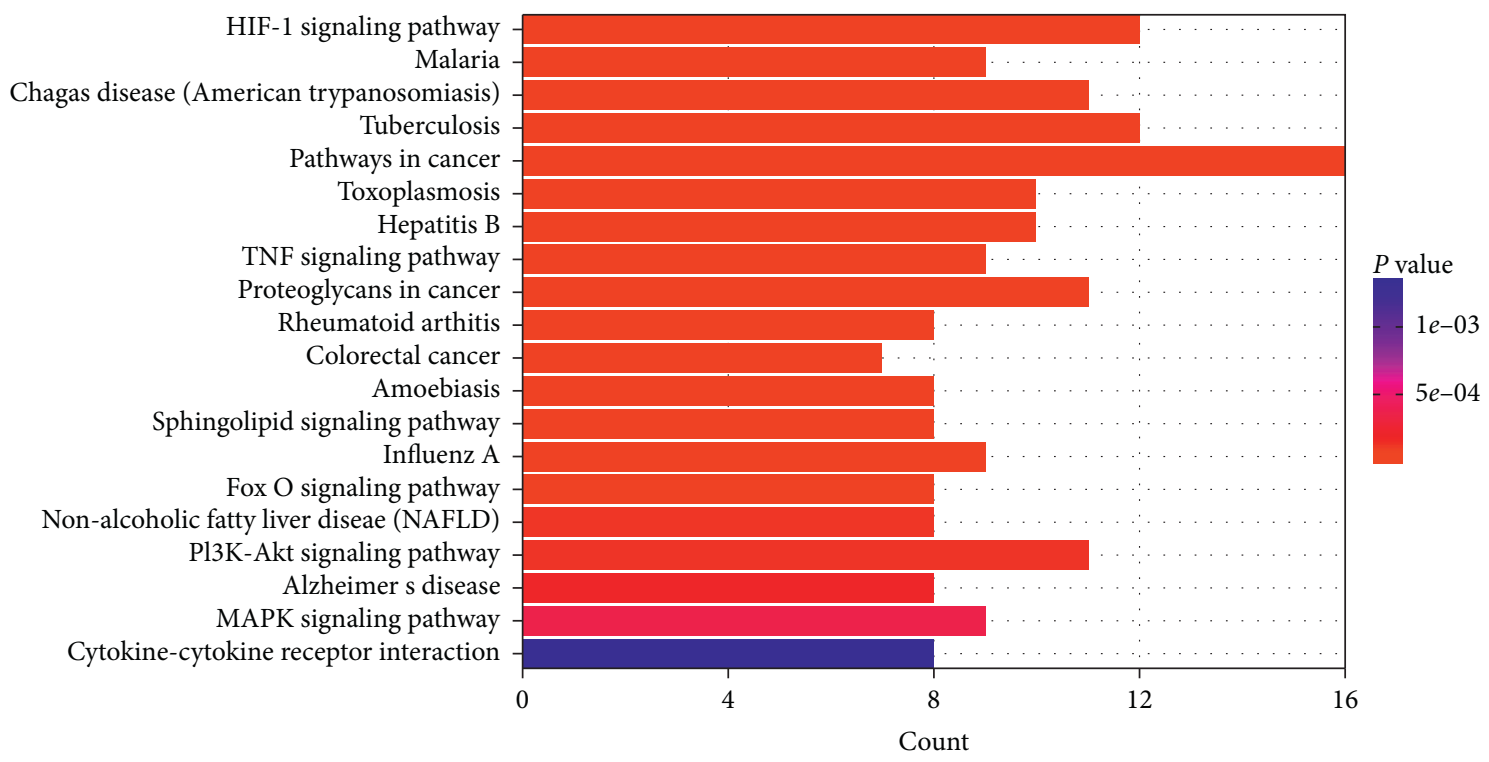

(a)

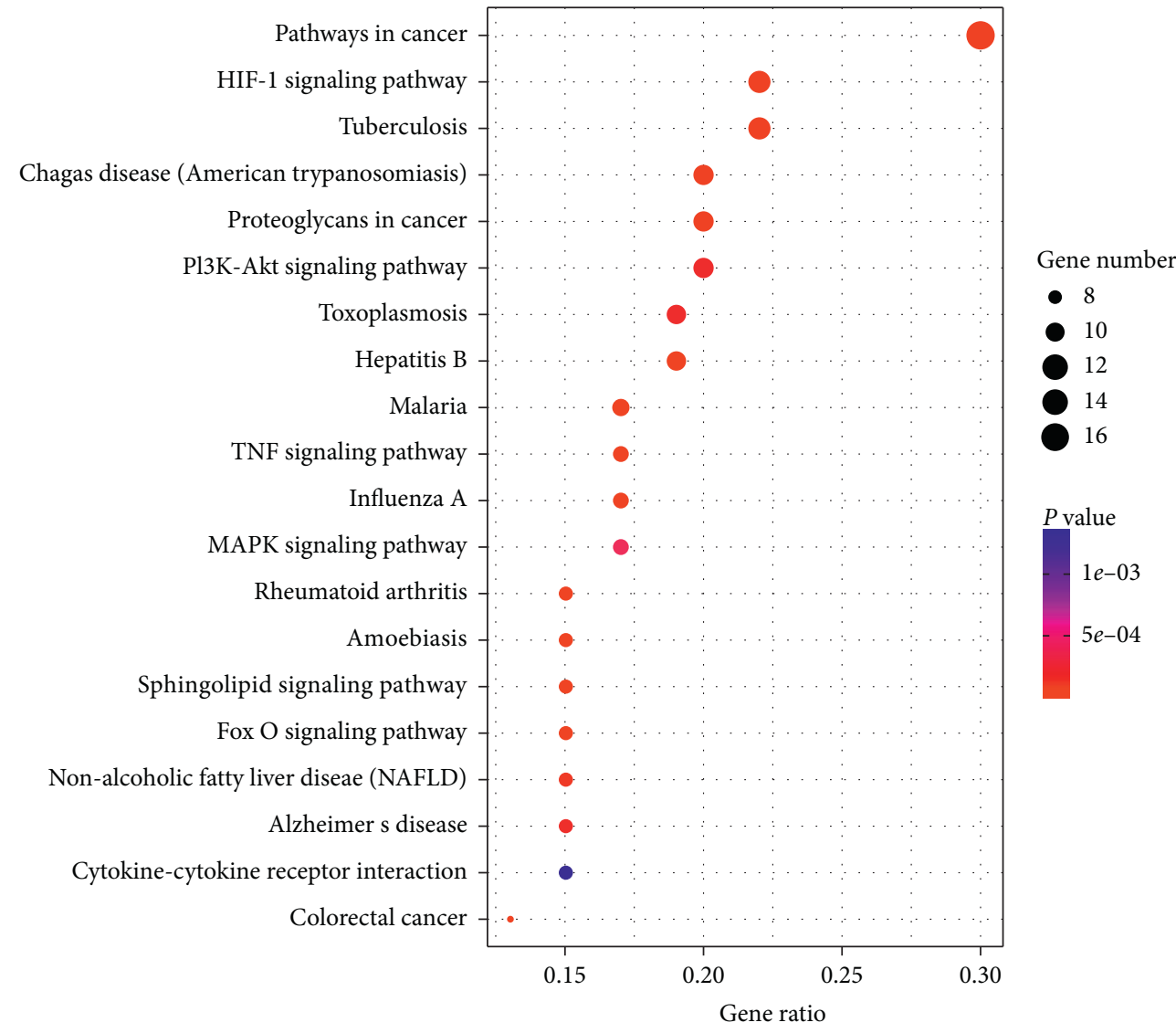

(b)

Figure 6: KEGG enrichment analysis of 54 target genes. (a) Barplot of KEGG analysis. (b) Dotplot of KEGG analysis: the size of dots indicates the number of target genes; the different color of dots indicates different $P$ value ranges.

likely to be "master regulators" and could be major mediators of neurodegenerative diseases. For example, AKT1 regulates cell survival, proliferation, and growth and metabolism mediated by serine and threonine phosphorylation of downstream substrates [41, 42]. AKT positively regulates CREB1 protein activity and plays a crucial role in the regulation of $\mathrm{NF}-\kappa \mathrm{B}$-dependent gene transcription. CREB1 phosphorylation induces the binding of auxiliary proteins that are also necessary for the transcription of prosurvival genes, such as BCL2 and MCL1 [43]. The MAPK/ 


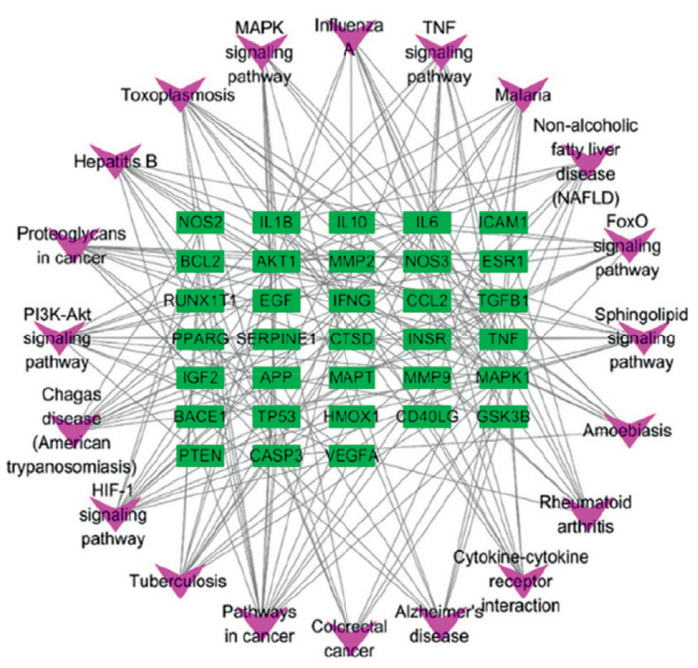

(a)

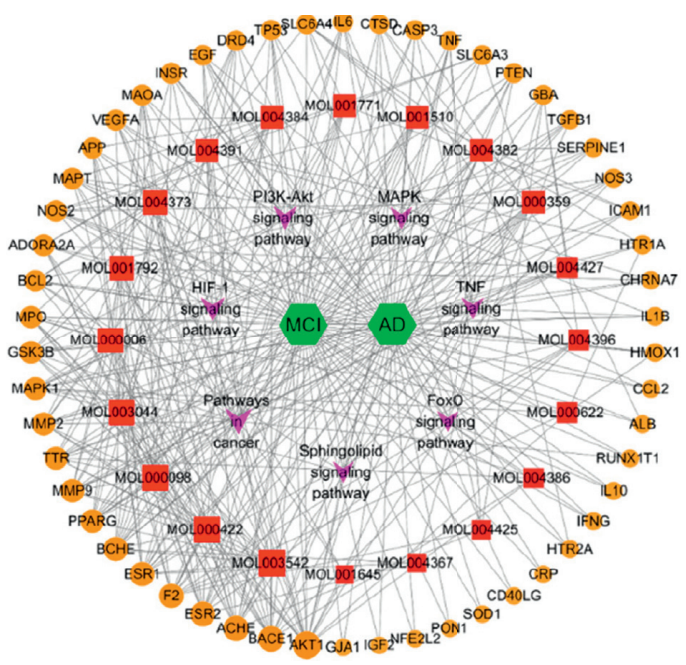

(b)

Figure 7: Analysis of targets-pathway network. (a) Network of 20 pathways and their shared targets: pathways were represented by arrowheads (purple), shared targets by rectangle (green). (b) An integral network of Epimedium active ingredients, MCI, and AD: Epimedium active ingredients were represented by square (red), disease by hexagons (green), target genes by circular (orange), and pathways by arrowheads (purple).

TABLE 3: The binding free energy of 20 small molecules with top 5 genes.

\begin{tabular}{|c|c|c|c|c|c|}
\hline Affinity $(\mathrm{kcal} / \mathrm{mol})$ & ALB & AKT1 & IL6 & CASP3 & TNF \\
\hline 24-epicampesterol & -9.3 & -7.7 & -6.5 & -7.1 & -7.1 \\
\hline Linoleyl acetate & -7.7 & -6.2 & -4.6 & -5.7 & -4.4 \\
\hline Poriferast-5-en-3beta-ol & -9.8 & -7.7 & -7.6 & -7.0 & -7.0 \\
\hline DFV & -7.8 & -7.6 & -7.0 & -6.9 & -6.1 \\
\hline Chryseriol & -9.0 & -8.5 & -7.1 & -7.4 & -6.5 \\
\hline 8-lsopentenyl-kaempferol & -8.4 & -8.5 & -6.9 & -7.8 & -6.8 \\
\hline Sitosterol & -9.7 & -8.9 & -7.4 & -7.4 & -7.4 \\
\hline Kaempferol & -8.6 & -8.0 & -6.8 & -7.2 & -6.5 \\
\hline Olivil & -8.5 & -8.6 & -6.3 & -7.1 & -6.5 \\
\hline Anhydroicaritin & -8.6 & -8.5 & -7.0 & -7.8 & -6.6 \\
\hline Yinyanghuo A & -10.0 & -9.7 & -6.7 & -8.4 & -8.0 \\
\hline Yinyanghuo $\mathrm{C}$ & -11.3 & -9.4 & -7.7 & -9.2 & -7.6 \\
\hline Yinyanghuo E & -11.1 & -9.6 & -7.6 & -9.3 & -7.3 \\
\hline 8-(3-methylbut-2-enyl)-2-phenyl-chromone & -10.5 & -9.2 & -7.2 & -7.6 & -7.3 \\
\hline 1,2-bis(4-hydroxy-3-methoxyphenyl) propane-1,3-diol & -7.9 & -7.5 & -7.0 & -6.2 & -6.2 \\
\hline Icariin & -9.2 & -9.3 & -6.8 & -8.2 & -6.6 \\
\hline Icariside A7 & -8.3 & -9.2 & -7.0 & -7.6 & -7.1 \\
\hline Luteolin & -9.4 & -8.5 & -7.1 & -7.8 & -6.7 \\
\hline Magnograndiolide & -7.8 & -8.2 & -6.4 & -6.8 & -6.1 \\
\hline Quercetin & -8.8 & -8.2 & -6.8 & -7.3 & -6.5 \\
\hline
\end{tabular}

ERK cascade accommodates biological functions such as cell survival, growth, adhesion, and differentiation and phosphorylates transcription factors and regulates meiosis and mitosis in differentiated cells [44].

BCL2 is a pivotal apoptosis regulator in the apoptotic signaling pathway. BCL2 inhibits apoptosis in the outer mitochondrial membrane and inhibits the reduction of glutathione and redox state, controls membrane potential, and prevents the release of pro-apoptotic proteins such as cytochrome $\mathrm{C}$ and apoptosis-inducing factor AIF to suppress apoptosis $[45,46]$. Caspase-3 (CASP3) is an important pro-apoptotic protease that effects apoptosis mediators such as BCL2 and Bax. BCL2 is a direct substrate upstream of
CASP3. BCL2 and CASP 3 are both interrelated and mutually constrained and are jointly involved in the process of neuronal apoptosis $[47,48]$. Interleukin-6 (IL6) is a cellular inflammatory factor secreted by monocytes and is involved in neuroinflammatory processes along with IL10 and IL1 $\beta$. IL6 exacerbates the expression of inflammatory factor IL10 in downstream signaling pathways and promotes the oxidative stress response, leading to apoptosis or atrophy of neuronal cells. Moreover, IL6 promotes $\beta$-amyloid deposition and affects the integrity of neuronal axons or dendritic membranes $[49,50]$.

The MAPK signaling pathway is one of the essential signaling systems in living organisms and has been broadly 


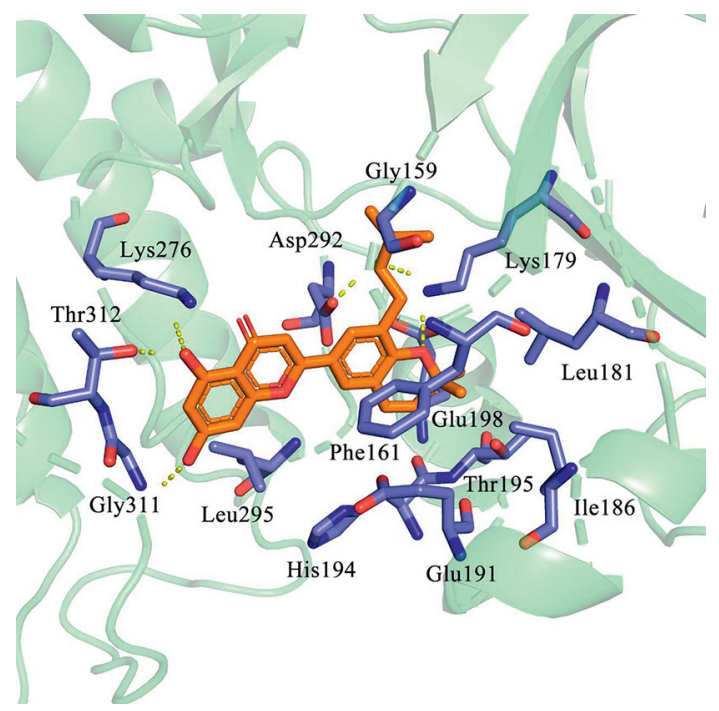

(a)

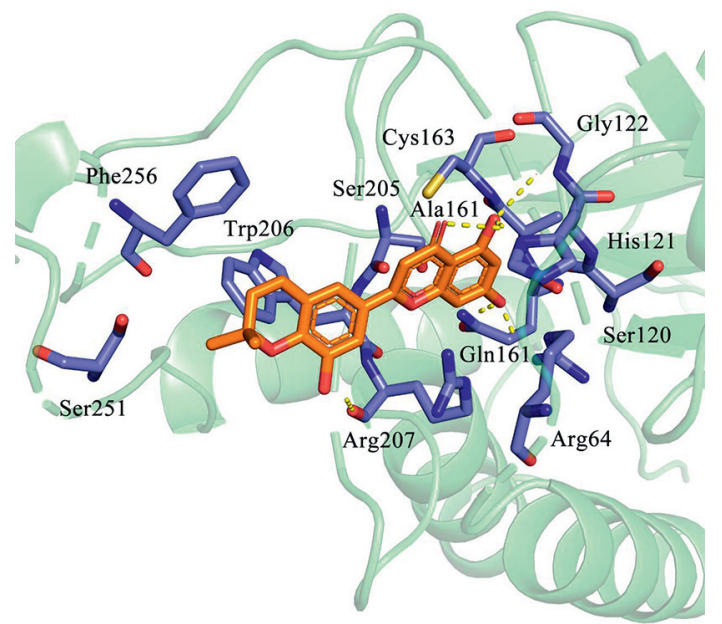

(c)

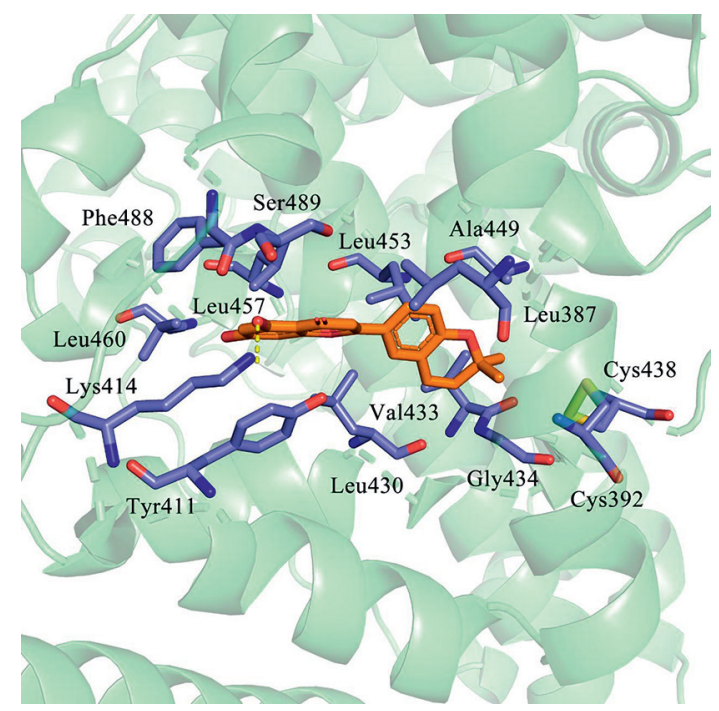

(b)

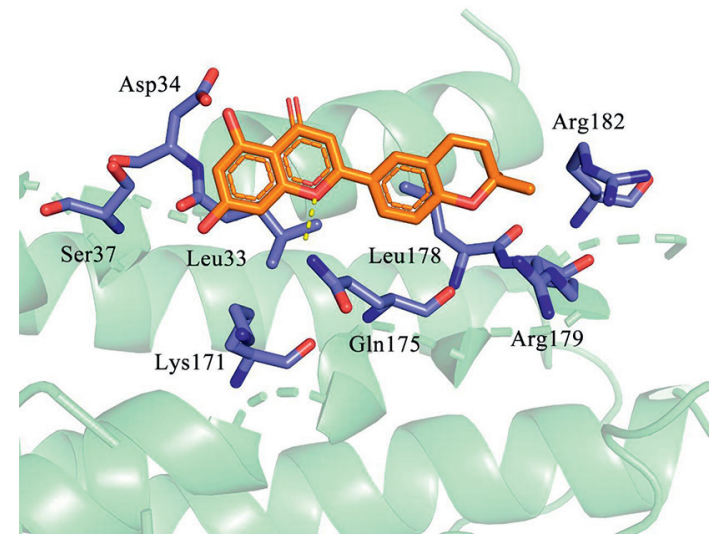

(d)

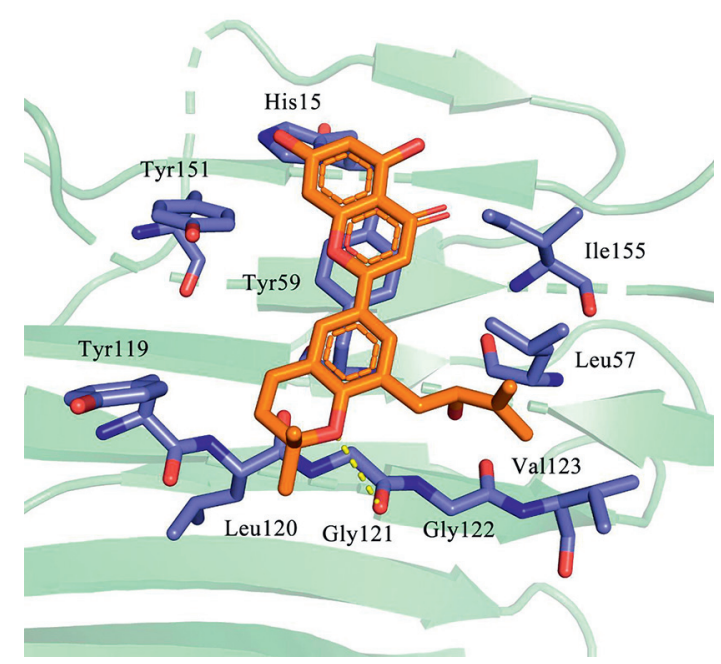

(e)

Figure 8: The molecular docking results of active ingredients and targets. (a) Binding pattern between Yinyanghuo A and AKT1. (b) Binding pattern between Yinyanghuo C and ALB. (c) Binding pattern between Yinyanghuo E and CASP3. (d) Binding pattern between Yinyanghuo $\mathrm{C}$ and IL6. (e) Binding pattern between Yinyanghuo A and TNF. 
studied in plants, insects, and mammals. MAPK signaling participates in a wide variety of physiopathological processes, such as cell growth, development, differentiation, and apoptosis, and is a regulator of inflammation and the stress response [51]. Neuroinflammation is the subject of recent attention as a latent element in the pathology of AD. Massive $\beta$-amyloid deposition and hyperphosphorylation of tau protein bring about cell autophagy, function loss, and apoptosis, and these pathological reactions are compactly associated with MAPK activation and signal conduction [52]. Pinocembrin, a natural flavonoid compound, inhibits RAGE expression both in vivo and in vitro, preventing P38MAPK and MK2 activation and downregulating the NF$\kappa \mathrm{B}$ and SAPK/JNK-c-Jun signaling pathways to reduce cerebral cortex neurodegeneration and ameliorate cognitive function [53, 54]. Drugs that directly target the MAPK signaling pathway may prove to be an effective strategy for the treatment of $\mathrm{AD}$.

The PI3K-Akt signaling pathway participates in the processes of cell reproduction, differentiation, and apoptosis and has many effectors downstream. Akt is the central link in the PI3K-Akt signaling pathway, and it translocates from the cell membrane to the nucleus, where it affects many transcriptional regulators and factors after activation, such as NF- $\kappa$ B, CREB, mTOR, GSK- $3 \beta$, caspase, and the BCL2 family [55]. The PI3K-Akt signaling pathway is involved in the energy metabolism of the aging process, as well as numerous physiological-pathological processes of age-related diseases. Polyphenolic anthocyanins are a natural compound with antioxidant and neuroprotective features that reduce the production of $\mathrm{A} \beta$ and ROS in in vitro $\mathrm{AD}$ models and APP/PS1 transgenic mouse models. Polyphenolic anthocyanins reduce oxidative stress to exert their neuroprotective effects, improve memory dysfunction, and delay neurodegenerative diseases through the regulation of the PI3K/Akt/Nrf2 pathway [56].

The HIF-1 signaling pathway is a physiological response to hypoxia that is mediated by hypoxia-inducible factors (HIFs) and activated or initiated in mammals to adapt to hypoxic environments, also allowing for the accommodation of hypoxia and increased cellular energy metabolism [57]. HIFs are involved in many disease processes, such as inflammation-induced cancer exacerbation, angiogenesis, malignant cell proliferation, and anti-apoptosis [58]. During aging, the supply of oxygen to the brain gradually decreases, and metabolism slows affecting APP expression and transport, leading to an imbalance between the production and clearance of $\mathrm{A} \beta$ and increasing the risk of $\mathrm{AD}$ [59]. Animal experiments suggest that HIF- $1 \alpha$ upregulates BACE1 expression and catalyzes $\beta$-cleavage of APP, while BACE1 expression in the hippocampus and cerebral cortex of mice is reduced by the lack of HIF- $1 \alpha$. The above examples demonstrate that the HIF-1 signaling pathway is one of the most important pathways for regulating APP amyloidosis [60].

The sphingolipid signaling pathway plays crucial roles in aging, neurodegenerative diseases, and immune regulation [61]. Sphingosine, sphingosine-1-phosphate, ceramide, and ceramide-1-phosphate are functionally similar and have modulatory effects on proliferation, differentiation, maturation, aging, death, stress response, and inflammation in central nervous cells and are currently the strongest known biologically active sphingomyelin compounds [62]. For example, A $\beta 25-35$ was applied to SH-SY5Y neuronal cells to create an AD model to induce SPHK1 inhibition and neuroamide accumulation, but the upregulation of sphingosine kinase prevented cell death and exerted a protective function [63]. Sphingolipids are pro-secretory molecules that regulate APP secretion and directly protect the nervous system of AD patients, which have excellent prospects for clinical application [64].

Forkhead box class $\mathrm{O}$ (FoxO) is a mammalian forkhead transcription factor that interferes with differentiated cell survival and stem cell proliferation and is a potential target for the control of neurological diseases [65]. The FoxO transcription factor interacts with numerous signaling pathways, such as Akt, SIRT1, IKK, and growth factor $[66,67]$. FoxO proteins are selectively expressed throughout the body and exert different biological functions in the nervous system [68]. The complex interactions between FoxO proteins and their signal transduction pathways are capable of promoting cellular autophagy, exerting antioxidant effects under oxidative stress, improving cell survival, and scavenging toxic proteins [69]. FoxO proteins hold promise as a new strategy for the treatment of neurodegenerative diseases.

Tumor necrosis factor (TNF) is a cytokine ligand that participates in systemic inflammation. Both TNF and its type 1 receptor (TNFRI) are involved in AD-associated cerebral neuroinflammation and regulate amyloid formation through secreted enzymes [70]. Aging, injury, or degenerative neurological conditions contribute to the production and release of TNF $\alpha$, inducing destructive or toxic biological effects when it binds to specific receptors [71]. Developing drugs to modulate the production of TNF $\alpha$, such as NF- $\kappa \mathrm{B}$, P38 MAPK, and TNF receptor antagonist inhibitors, may be potential candidates for the treatment of cognitive impairment, $\mathrm{AD}$, and other neurodegenerative diseases.

As mentioned above, the targets identified in our study are consistent with previous attempts. Epimedium active ingredients have confirmed interacting partners with proteins that are important regulators of health and disease; these interactions could point future research towards novel therapeutic strategies for treating neurodegenerative diseases.

\section{Conclusions}

In summary, we made use of network pharmacology to reveal the interactions between the possible active ingredients of Epimedium and known therapeutic genes associated with $\mathrm{MCI}$ and $\mathrm{AD}$. Through further screening, we verified the potential targets of Epimedium at the molecular level and predicted possible major regulatory pathways that are involved in its neuroprotective effects. The pathogenesis of $\mathrm{MCI}$ and $\mathrm{AD}$ is complicated, forming a multi-path and multi-target action mode with interlaced signal pathways. The active ingredients of Epimedium against $\mathrm{MCI}$ and $\mathrm{AD}$ 
may function by modulating these pathways, demonstrating the multi-component and multi-target capabilities of Chinese herbs. Our findings provide a hypothetical basis for further practical molecular studies of the interactions between Epimedium and these proteins. The combination of Chinese medicine and modern analytical methods will allow in-depth studies of Epimedium's efficacy at treating neurodegenerative diseases. Our findings could lay the foundation for pragmatic clinical application of Epimedium, and new drug development.

\section{Data Availability}

The data used in the study are available upon request to the corresponding author.

\section{Conflicts of Interest}

All authors have no conflicts of interest to declare.

\section{Authors' Contributions}

XWG, SNL, CC, YJW, and LWX proposed the concept and design of the study. XWG and SNL contributed to collecting and analyzing data. CC and YJW contributed to checking the accuracy of data. XWG and CC drafted the main manuscript text, while SNL, YJW, and LWX contributed to editing and providing critical revision. All the authors reviewed and approved the final manuscript.

\section{Acknowledgments}

This study was supported by the National Natural Science Foundation of China (No. 81874482).

\section{Supplementary Materials}

The characteristics of 130 chemical ingredients in Epimedium are shown in Supplementary Table 1. The compoundrelated targets were selected from TCMSP; SwissTarget and PharmMapper are displayed in Supplementary Table 2. The acknowledged targets of mild cognitive impairment and Alzheimer's disease are listed in Supplementary Table 3. The detailed information about GO/KEGG enrichment analysis is presented in Supplementary Tables 4 and 5. (Supplementary Materials)

\section{References}

[1] M. S. Uddin, M. T. Kabir, K. Niaz et al., "Molecular insight into the therapeutic promise of flavonoids against Alzheimer's disease," Molecules, vol. 25, no. 6, p. 1267, 2020.

[2] M. S. Uddin, M. T. Kabir, M. S. Rahman et al., "Revisiting the amyloid cascade hypothesis: from anti- $\mathrm{A} \beta$ therapeutics to auspicious new ways for Alzheimer's disease," International Journal of Molecular Sciences, vol. 21, no. 16, p. 5858, 2020.

[3] M. F. Hossain, M. S. Uddin, G. M. S. Uddin et al., "Melatonin in Alzheimer's disease: a latent endogenous regulator of neurogenesis to mitigate Alzheimer's neuropathology," Molecular Neurobiology, vol. 56, no. 12, pp. 8255-8276, 2019.
[4] P. Sharma, A. Sharma, F. Fayaz, S. Wakode, and F. H. Pottoo, "Biological signatures of Alzheimer's disease," Current Topics in Medicinal Chemistry, vol. 20, no. 9, pp. 770-781, 2020.

[5] M. A. Rahman, M. R. Rahman, T. Zaman et al., "Emerging potential of naturally occurring autophagy modulators against neurodegeneration," Current Pharmaceutical Design, vol. 26, no. 7, pp. 772-779, 2020.

[6] M. T. Kabir, M. S. Uddin, A. Abdeen et al., "Evidence linking protein misfolding to quality control in progressive neurodegenerative diseases," Current Topics in Medicinal Chemistry, vol. 20, no. 23, pp. 2025-2043, 2020.

[7] M. S. Uddin, D. Tewari, A. A. Mamun et al., "Circadian and sleep dysfunction in Alzheimer's disease," Ageing Research Reviews, vol. 60, p. 101046, 2020.

[8] A. L. Sosa-Ortiz, I. Acosta-Castillo, and M. J. Prince, "Epidemiology of dementias and Alzheimer's disease," Archives of Medical Research, vol. 43, no. 8, pp. 600-608, 2012.

[9] V. V. Giau, E. Bagyinszky, and S. S. An, "Potential fluid biomarkers for the diagnosis of mild cognitive impairment," International Journal of Molecular Sciences, vol. 20, no. 17, p. 4149, 2019.

[10] M. S. An, S. T. DeKosky, D. Dickson et al., "The diagnosis of mild cognitive impairment due to Alzheimer's disease: recommendations from the National Institute on Aging-Alzheimer's Association workgroups on diagnostic guidelines for Alzheimer's disease," Alzheimer's \& Dementia, vol. 7, no. 3, pp. 270-279, 2011.

[11] N. D. Anderson, "State of the science on mild cognitive impairment (MCI)," CNS Spectrums, vol. 24, no. 1, pp. 78-87, 2019.

[12] Alzheimer's Association, “Alzheimer's disease facts and figures," Alzheimers Dement, vol. 16, pp. 391-460, 2020.

[13] M. T. Kabir, M. S. Uddin, A. A. Mamun et al., "Combination drug therapy for the management of Alzheimer's disease," International Journal of Molecular Sciences, vol. 21, no. 9, p. 3272, 2020.

[14] E. Nichols, C. E. I. Szoeke, S. E. Vollset et al., "Global, regional, and national burden of Alzheimer's disease and other dementias, 1990-2016: a systematic analysis for the global burden of disease study 2016," Lancet Neurology, vol. 18, no. 5, pp. 459-480, 2019.

[15] S. C. W. Sze, Y. Tong, T. B. Ng, C. L. Y. Cheng, and H. P. Cheung, "Herba epimedii: anti-oxidative properties and its medical implications," Molecules, vol. 15, no. 11, pp. 7861-7870, 2010.

[16] Z. Wei, M. Wang, M. Hong et al., "Icariin exerts estrogen-like activity in ameliorating EAE via mediating estrogen receptor $\beta$, modulating HPA function and glucocorticoid receptor expression," American Journal of Translational Research, vol. 8, no. 4, pp. 1910-1918, 2016.

[17] Y. L. Lan, J. Zhao, and S. Li, "Update on the neuroprotective effect of estrogen receptor alpha against Alzheimer's disease," Journal of Alzheimer's Disease: JAD, vol. 43, no. 4, pp. 1137-1148, 2015.

[18] Chinese Pharmacopoeia Commission, Pharmacopoeia of the People's Republic of China, China Medical Science Press, Beijing, China, 2015.

[19] X.-J. Chen, Z.-H. Tang, X.-W. Xie, J.-J. Lu, and Y.-T. Wang, "Chemical constituents, quality control, and bioactivity of Epimedii folium (Yinyanghuo)," The American Journal of Chinese Medicine, vol. 43, no. 05, pp. 783-834, 2015.

[20] I. R. Indran, R. L. Z. Liang, T. E. Min, and E.-L. Yong, "Preclinical studies and clinical evaluation of compounds 
from the genus epimedium for osteoporosis and bone health," Pharmacology \& Therapeutics, vol. 162, pp. 188-205, 2016.

[21] L. Zhang, C. Shen, J. Chu, R. Zhang, Y. Li, and L. Li, "Icariin decreases the expression of APP and BACE-1 and reduces the $\beta$-amyloid burden in an APP transgenic mouse model of Alzheimer's disease," International Journal of Biological Sciences, vol. 10, no. 2, pp. 181-191, 2014.

[22] D. Zhang, Z. Wang, and C. Sheng, "Icariin prevents amyloid beta-induced apoptosis via the PI3K/Akt pathway in PC-12 cells," Evidence-Based Complementary and Alternative Medicine, vol. 2015, Article ID 235265, 9 pages, 2015.

[23] S. Yue, L. Xin, Y. Fan et al., "Herb pair Danggui-Honghua: mechanisms underlying blood stasis syndrome by system pharmacology approach," Scientific Reports, vol. 7, p. 40318, 2017.

[24] C. Lu, Z. Bing, Z. Bi et al., "Top-100 most cited publications concerning network pharmacology: a bibliometric analysis," Evidence-Based Complementary and Alternative Medicine, vol. 2019, Article ID 1704816, 7 pages, 2019.

[25] J. Ru, L. Peng, W. Jinan et al., "TCMSP: a database of systems pharmacology for drug discovery from herbal medicines," Journal of Cheminformatics, vol. 6, no. 1, p. 13, 2014.

[26] S. Kim, P. A. Thiessen, E. E. Bolton et al., "PubChem substance and compound databases," Nucleic Acids Research, vol. 44, no. D1, pp. D1202-D1213, 2016.

[27] D. Gfeller, A. Grosdidier, M. Wirth, A. Daina, O. Michielin, and V. Zoete, "SwissTargetPrediction: a web server for target prediction of bioactive small molecules," Nucleic Acids Research, vol. 42, no. W1, pp. W32-W38, 2014.

[28] X. Wang, Y. Shen, S. Wang et al., "PharmMapper 2017 update: a web server for potential drug target identification with a comprehensive target pharmacophore database," Nucleic Acids Research, vol. 45, no. W1, pp. W356-W360, 2017.

[29] The UniProt Consortium, "UniProt: the universal protein knowledge base," Nucleic Acids Research, vol. 46, no. 5, pp. D158-D169, 2017.

[30] M. Safran, I. Dalah, J. Alexander et al., "GeneCards version 3: the human gene integrator," Database (Oxford), vol. 2010, Article ID baq020, 16 pages, 2010.

[31] J. Piñero, À. Bravo, N. Queralt-Rosinach et al., "DisGeNET: a comprehensive platform integrating information on human disease-associated genes and variants," Nucleic Acids Research, vol. 45, no. D1, pp. D833-D839, 2017.

[32] J. Amberger, C. A. Bocchini, A. F. Scott, and A. Hamosh, "McKusick's online mendelian inheritance in man (OMIM(R))," Nucleic Acids Research, vol. 37, no. Database, pp. D793-D796, 2009.

[33] D. Szklarczyk, J. H. Morris, H. Cook et al., "The STRING database in 2017: quality-controlled protein-protein association networks, made broadly accessible," Nucleic Acids Research, vol. 45, no. D1, pp. D362-D368, 2017.

[34] D. W. Huang, B. T. Sherman, and R. A. Lempicki, "Systematic and integrative analysis of large gene lists using DAVID bioinformatics resources," Nature Protocols, vol. 4, no. 1, pp. 44-57, 2009.

[35] P. W. Rose, A. Prlić, A. Altunkaya et al., "The RCSB protein data bank: integrative view of protein, gene and 3D structural information," Nucleic Acids Research, vol. 45, no. D1, pp. D271-D281, 2017.

[36] F. Li, H. X. Dong, Q. H. Wu, F. Jin, and J. S. Shi, "Icariin decreases both APP and A $\beta$ levels and increases neurogenesis in the brain of Tg2576 mice," Neuroscience, vol. 304, pp. 29-35, 2015.
[37] Y.-J. Chen, H.-Y. Zheng, X.-X. Huang et al., "Neuroprotective effects of icariin on brain metabolism, mitochondrial functions, and cognition in triple-transgenic Alzheimer's disease mice," CNS Neuroscience \& Therapeutics, vol. 22, no. 1, pp. 63-73, 2016.

[38] P. Babaei, S. Kouhestani, and A. Jafari, "Kaempferol attenuates cognitive deficit via regulating oxidative stress and neuroinflammation in an ovariectomized rat model of sporadic dementia," Neural Regeneration Research, vol. 13, no. 10, pp. 1827-1832, 2018.

[39] E. Zaplatic, M. Bule, S. Z. A. Shah, M. S. Uddin, and K. Niaz, "Molecular mechanisms underlying protective role of quercetin in attenuating Alzheimer's disease," Life Sciences, vol. 224, pp. 109-119, 2019.

[40] M. A. Ansari, H. M. Abdul, G. Joshi, W. O. Opii, and D. A. Butterfield, "Protective effect of quercetin in primary neurons against $\mathrm{A} \beta(1-42)$ : relevance to Alzheimer's disease," The Journal of Nutritional Biochemistry, vol. 20, no. 4, pp. 269-275, 2009.

[41] L. H. Milhavet, N. Khouya, A. Fernandez, and N. J. Lamb, "Akt1 and Akt2: differentiating the aktion," Histology and histopathology, vol. 26, no. 5, pp. 651-662, 2011.

[42] I. Hers, E. E. Vincent, and J. M. Tavaré, "Akt signalling in health and disease," Cellular Signalling, vol. 23, no. 10, pp. 1515-1527, 2011.

[43] K. Du and M. Montminy, "CREB is a regulatory target for the protein kinase Akt/PKB," Journal of Biological Chemistry, vol. 273, no. 49, pp. 32377-32379, 1998.

[44] Y. Sun, W.-Z. Liu, T. Liu, X. Feng, N. Yang, and H.-F. Zhou, "Signaling pathway of MAPK/ERK in cell proliferation, differentiation, migration, senescence and apoptosis," Journal of Receptors and Signal Transduction, vol. 35, no. 6, pp. 600-604, 2015.

[45] P. Theofilas, P. Bedner, K. Hüttmann, M. Theis, C. Steinhäuser, and S. Frank, "The proapoptotic BCL-2 homology domain 3-only protein bim is not critical for Acute excitotoxic cell death," Journal of Neuropathology \& Experimental Neurology, vol. 68, no. 1, pp. 102-110, 2009.

[46] S. L. B. Haeberlein, "Mitochondrial function in apoptotic neuronal cell death," Neurochemical Research, vol. 29, no. 3, pp. 521-530, 2004.

[47] C. A. Odonkor and S. Achilefu, "Modulation of effector caspase cleavage determines response of breast and lung tumor cell lines to chemotherapy," Cancer Investigation, vol. 27, no. 4, pp. 417-429, 2009.

[48] L. Scorrano and S. J. Korsmeyer, "Mechanisms of cytochrome c release by proapoptotic BCL-2 family members," Biochemical and Biophysical Research Communications, vol. 304, no. 3, pp. 437-444, 2003.

[49] H. K. A. Shin, "Differences in C-reactive protein level in patients with Alzheimers disease and mild cognitive impairment," African Jo Psychiatry, vol. 18, no. 1, 2015.

[50] C. R. Gelman, "Familismo and its impact on the family caregiving of Latinos with Alzheimer's disease: a complex narrative," Research on Aging, vol. 36, no. 1, pp. 40-71, 2014.

[51] R. K. C. Venigalla and M. Turner, "RNA-binding proteins as a point of convergence of the PI3 K and p38 MAPK pathways," Frontiers in Immunology, vol. 3, p. 398, 2012.

[52] G. Kheiri, M. Dolatshahi, F. Rahmani, and N. Rezaei, "Role of p38/MAPKs in Alzheimer's disease: implications for amyloid beta toxicity targeted therapy," Reviews in the Neurosciences, vol. 30, no. 1, pp. 9-30, 2018.

[53] Z.-H. Yang, X. Sun, Y. Qi, C. Mei, X.-B. Sun, and G.-H. Du, "Uptake characteristics of pinocembrin and its effect on 
p-glycoprotein at the blood-brain barrier inin vitrocell experiments," Journal of Asian Natural Products Research, vol. 14, no. 1, pp. 14-21, 2012.

[54] R. Liu, C. X. Wu, D. Zhou et al., "Pinocembrin protects against beta amyloid-induced toxicity in neurons through inhibiting receptor for advanced glycation end products (RAGE)-independent signaling pathways and regulating mitochondrionmediated apoptosis," BMC Medicine, vol. 10, p. 105, 2012.

[55] A. K. Singh, M. P. Kashyap, V. K. Tripathi, S. Singh, G. Garg, and S. I. Rizvi, "Neuroprotection through rapamycin-induced activation of autophagy and PI3K/Akt1/mTOR/CREB signaling against amyloid- $\beta$-induced oxidative stress, synaptic/ neurotransmission dysfunction, and neurodegeneration in Adult rats," Molecular Neurobiology, vol. 54, no. 8, pp. 5815-5828, 2017.

[56] T. Ali, T. Kim, S. U. Rehman et al., "Natural dietary supplementation of anthocyanins via PI3K/Akt/Nrf2/HO-1 pathways mitigate oxidative stress, neurodegeneration, and memory impairment in a mouse model of Alzheimer's disease," Molecular Neurobiology, vol. 55, no. 7, pp. 6076-6093, 2018.

[57] C. Takasaki, M. Kobayashi, H. Ishibashi, T. Akashi, and K. Okubo, "Expression of hypoxia-inducible factor- $1 \alpha$ affects tumor proliferation and antiapoptosis in surgically resected lung cancer," Molecular and Clinical Oncology, vol. 5, no. 2, pp. 295-300, 2016.

[58] S. Neitemeier, A. M. Dolga, B. Honrath et al., "Inhibition of HIF-prolyl-4-hydroxy lases prevents mitochondrial impairment and cell death in a model of neuronal oxytosis," Cell Death \& Disease, vol. 7, no. 5, Article ID e2214, 2016.

[59] I. L. Ferreira, R. Resende, E. Ferreiro, A. C Rego, and C. F Pereira, "Multiple defects in energy metabolism in Alzheimer's disease," Current Drug Targets, vol. 11, no. 10, pp. 1193-1206, 2010.

[60] X. Zhang, K. Zhou, R. Wang et al., "Hypoxia-inducible factor $1 \alpha(\mathrm{HIF}-1 \alpha)$-mediated hypoxia increases BACE1 expression and $\beta$-amyloid generation," Journal of Biological Chemistry, vol. 282, no. 15, pp. 10873-10880, 2007.

[61] S. Nakamura, E. Fukai, S. Miya et al., "Sphingolipid signaling and neuronal function," Chemistry and Physics of Lipids, vol. 164, no. S9, 2011.

[62] M. Maceyka and S. Spiegel, "Sphingolipid metabolites in inflammatory disease," Nature, vol. 510, no. 7503, pp. 58-67, 2014.

[63] A. G. Brouchet, D. Pchejetski, L. Brizuela et al., "Critical role for sphingosine kinase-1 in regulating survival of neuroblastoma cells exposed to amyloid-beta peptide," Molecular Pharmacology, vol. 72, no. 2, pp. 341-349, 2007.

[64] H. Jesko, T. Okada, R. P. Strosznajder, and S.-i. Nakamura, "Sphingosine kinases modulate the secretion of amyloid $\beta$ precursor protein from SH-SY5Y neuroblastoma cells: the role of $\alpha$-synuclein," Folia Neuropathologica, vol. 1, pp. 70-78, 2014.

[65] D. Weigel, G. Jürgens, F. Küttner, E. Seifert, and H. Jäckle, "The homeotic gene fork head encodes a nuclear protein and is expressed in the terminal regions of the drosophila embryo," Cell, vol. 57, no. 4, pp. 645-658, 1989.

[66] M. C.-T. Hu, D.-F. Lee, W. Xia et al., "I $\kappa B$ kinase promotes tumorigenesis through inhibition of forkhead FOXO3a," Cell, vol. 117, no. 2, pp. 225-237, 2004.

[67] A. van der Horst and B. M. T. Burgering, "Stressing the role of FoxO proteins in lifespan and disease," Nature Reviews Molecular Cell Biology, vol. 8, no. 6, pp. 440-450, 2007.
[68] S. Peng, S. Zhao, F. Yan et al., "HDAC2 selectively regulates FOXO3a-mediated gene transcription during oxidative stressinduced neuronal cell death," Journal of Neuroscience, vol. 35, no. 3, pp. 1250-1259, 2015.

[69] K. Maiese, "FoxO proteins in the nervous system," Analytical Cellular Pathology, vol. 2015, Article ID 569392, 15 pages, 2015.

[70] P. He, Z. Zhong, K. Lindholm et al., "Deletion of tumor necrosis factor death receptor inhibits amyloid $\beta$ generation and prevents learning and memory deficits in Alzheimer's mice," Journal of Cell Biology, vol. 178, no. 5, pp. 829-841, 2007.

[71] X. Cheng, Y. Shen, and R. Li, "Targeting TNF: a therapeutic strategy for Alzheimer's disease," Drug Discovery Today, vol. 19, no. 11, pp. 1822-1827, 2014. 